\title{
COMPUTATIONAL INTELLIGENCE BASED SIMULTANEOUS DETERMINATION OF THE SPATIAL PROFILE OF THE LASER BEAM AND VIBRATIONAL-TO-TRANSLATIONAL RELAXATION TIME BY PULSED PHOTOACOUSTICS ${ }^{\dagger}$
}

\author{
$U D C 007.52+53.082 .4: 621.375 .826$
}

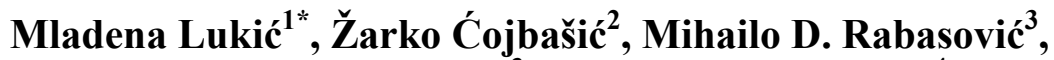 \\ Dragan D. Markushev ${ }^{3}$, Dragan M. Todorović ${ }^{4}$ \\ ${ }^{1}$ Faculty of Occupational Safety, University of Niš, Čarnojevića 10a, 18000 Niš, Serbia \\ ${ }^{2}$ Mechanical Engineering Faculty, University of Niš, Aleksandra Medvedeva 14, \\ $18000 \mathrm{Nišs}$, Serbia \\ ${ }^{3}$ Institute of Physics, University of Belgrade, Pregrevica 118, 11080 Beograd-Zemun, Serbia \\ ${ }^{4}$ Institute for Multidisciplinary Research, University of Belgrade, P.O. Box 33,
}

11030 Belgrade, Serbia

\begin{abstract}
This paper is concerned with the possibilities of computational intelligence application for simultaneous determination of the laser beam spatial profile and vibrational-to-translational relaxation time of the polyatomic molecules in gases by pulsed photoacoustics. Results regarding the application of neural computing and genetic optimization are presented through the use of feed forward multilayer perception networks and real-coded genetic algorithms. Feed forward multilayer perception networks are trained in an offline batch training regime to estimate simultaneously, and in real-time, laser beam spatial profile $R(r)$ (profile shape class) and vibrational-to-translational relaxation time $\tau_{\mathrm{V}-\mathrm{T}}$ from a given (theoretical) photoacoustic signals $\delta p(r, t)$. The proposed method significantly shortens the time required for the simultaneous determination of the laser beam spatial profile and relaxation time and has the advantage of accurately calculating the aforementioned quantities. Real coded genetic algorithms are used to calculate $\tau_{\mathrm{V}-\mathrm{T}}$ by fitting the $\delta p(r, t)$ with the theoretical one. The previously developed methods determine the laser beam profile and relaxation time with sufficient precision, but the methods based on the application of artificial intelligence are more suitable for practical applications, such as the real-time in-situ measurements of atmospheric pollutants.
\end{abstract}

Key words: photoacoustic spectroscopy, laser beam profile, vibrational-to-translational relaxation time, neural networks, genetic algorithms

Received August 22 $2^{\text {nd }}, 2011$, accepted January $19^{\text {th }}, 2012$.

${ }^{\dagger}$ Acknowledgement: This research was supported by the Ministry of Education and Science of the Republic of Serbia within the frame of projects No. 171016 and No. TR035016.

${ }^{*}$ Corresponding author. E-mail: mladena.lukic@znrfak.ni.ac.rs 


\section{INTRODUCTION}

In recent years air pollution has become an issue of worldwide concern. It has been recognized that even trace concentrations of atmospheric species (different type of molecules) can have a substantial impact in diverse areas. The well-known phenomena such as global warming, climatic change, acid rain, smog formation etc., are strongly related to increasing concentrations of trace species. The large number of species and diversity of phenomena in the atmosphere pose a great challenge with regard to a through understanding of the complex chemical and physical processes and interactions involved.

Molecules in the atmosphere usually gain and lose vibrational and rotational energy interacting with the radiation and/or in collisions. All of them can be activated or deactivated by absorption or emission of radiation, but the radiative lifetime is so long for infrared frequencies that this mechanism may be neglected under most conditions. In this respect the knowledge of the kind of species and of their infrared absorption and nonradiative (collisionally induced) relaxation characteristics in the atmosphere is an irrevocable prerequisite.

The nature of molecular nonradiative relaxation mechanisms is of fundamental importance if one wants to understand the physical and chemical kinetics in the atmosphere. This relaxation is related to some energy transfer processes between different modes of molecule (usually from vibrational to translational modes). There are three main nonradiative energy transfer processes: Vibrational-to-Translational (V-T), Rotational-toTranslational (R-T) and Vibrational-to-Vibrational $(\mathrm{V}-\mathrm{V})$ relaxation. Semiclassical and quantum mechanical theories predict that all mentioned energy transfer processes play an equally important role in vibrational relaxation. The average time required for establishment of equilibrium between two modes during the energy transfer process is, so called, relaxation time $\tau$. For all mentioned processes we can define different relaxation times: $\tau_{\mathrm{V}-\mathrm{T}}, \tau_{\mathrm{R}-\mathrm{T}}$ and $\tau_{\mathrm{V}-\mathrm{V}}$ respectively. Theoretical and experimental results show that $\mathrm{V}-\mathrm{T}$ is the slowest $\left(\tau_{\mathrm{V}-\mathrm{T}}>\tau_{\mathrm{R}-\mathrm{T}}>\tau_{\mathrm{V}-\mathrm{V}}\right)$ but dominant process in absorbed energy transfer to translational modes of the polyatomic molecules. Knowing $\tau_{\mathrm{V}-\mathrm{T}}$ allows one to calculate most important physical parameters of the molecule, such as: energy transfer collisional rate, energy transfer probability, collisional cross-section, etc. On the other hand, values of $\tau_{\mathrm{V}-\mathrm{T}}$ could be used as a basic parameters for different models of energy transfer in the atmosphere responsible for global warming and climate change.

Various spectroscopy methods and techniques have been developed and successfully applied to trace gas monitoring. Some of them have become standard methods and are widely used on a routine basis. In more recent time photoacoustic spectroscopy (PAS) technique have attracted considerable interest. It is a well known fact that PAS is based on the photoacoustic effect refers to the generation of acoustic waves due to the interaction of light with an absorbing medium. The characteristics of this acoustic waves are determined by the medium type of their propagation (gas in our case) and sound source temporal (molecular relaxation) and spatial (radiation source profile) characteristics. Also, it offers some unique features that are relevant for the air monitoring, e.g., high sensitivity and selectivity, large dynamic range, good temporal resolution, easiness of operation and field applicability.

The main quantity that can be measured in PAS is the photoacoustic (PA) signal, namely its shape and intensity. As we said earlier, V-T processes between the molecules (absorbing species) for given conditions are characterized by vibrational-to-translational relaxation time $\tau_{\mathrm{V}-\mathrm{T}}$. One of the first applications of PAS was the $\tau_{\mathrm{V}-\mathrm{T}}$ measurement. 
Within the frame of proposed methods $\tau_{\mathrm{V}-\mathrm{T}}$ can be calculated from the temporal shape of the measured acoustic signal. Beck and Gordon used the ratio of the minimum and maximum of the PA signals to calculate relaxation time, because the relaxation time is a single-valued function of this ratio $[1,2]$. Such method is simple, but inaccurate, because not whole, but only two points of the signal (minimum and maximum) are used for comparison between theoretical and experimental signals. The method which takes into account all points of the photoacoustic signal is presented in [3]. To implement this method, it is necessary to achieve a better matching between the experimental and theoretical signal. In our previous research it was concluded that exact knowledge of the laser beam (radiation source) spatial profile is crucial for the analysis of photoacoustic signal temporal shapes and $\tau_{\mathrm{V}-\mathrm{T}}$ calculations [3-5]. The laser beam spatial profiles are currently measured using different commercial instruments, but despite providing high resolution beam profiles, these devices cannot work with high power lasers [6]. Different numerical methods can be used for measurement of the laser beam spatial profile for a wide range of laser fluencies. Mathematical algorithm developed for photoacoustic tomography (PAT) is convenient to calculate the spatial part of energy density, which is directly related to the laser beam spatial profile [7]. This algorithm was used for simultaneous determination of the laser beam spatial profile and relaxation time, providing higher accuracy then one using the minimum and maximum of the PA signals [3]. Regardless of the high precision and exact reconstruction of the laser beam spatial profiles, the usage of these methods is limited due to the long computational time [8]. Because of this all methods mentioned here do not work in real-time but allows one to use them for the additional beam-profile post-correction procedure of experimental results.

In this paper, we present two different methods for simultaneous calculation of the laser beam spatial profile and $\tau_{\mathrm{V}-\mathrm{T}}$ values using artificial neural networks (ANNs) and genetic algorithms (GAs). Feed forward multilayer perception networks, applied to simultaneous determination of laser beam profile and relaxation time, provide solution in realtime and with high precision. Using this method, one can overcome the problem of possible unwanted variations in the used laser beam spatial profile during the two consecutive pulses, which can significantly influence the relaxation time calculations. On the other hand, application of real coded genetic algorithms (GA), although it does not solve the problem in real time, allows for combinations of photoacoustic signal parameters $\left(\tau_{\mathrm{V}-\mathrm{T}}\right.$ and $r_{\mathrm{L}}$ ) to be found, that provide the best match with the given PA signal efficiently and with good precision.

\section{THEORETICAL BACKGROUND}

There are several mechanisms for acoustic wave generation in medium, but in our case dominant mechanism is thermoelastic, caused by the pressure changes in gas. These perturbations in pressure must be small, so that we can use linearized wave equation. The shape of an acoustic wave generated by a given energy source can be calculated by solving the nonhomogeneous linearized wave equation:

$$
\frac{\partial^{2} \delta p(\mathbf{r}, t)}{\partial t^{2}}-c^{2} \nabla^{2} \delta p(\mathbf{r}, t)=S(\mathbf{r}, t)
$$


where $\delta p(\mathbf{r}, t)$ is the pressure discrepancy from its equilibrium state value, $c$ is the speed of sound and $S(\mathbf{r}, t)$ is the source function. If the distribution of excited molecules at location $r$ and time $t$ are given by some energy function $\mathrm{E}(\mathbf{r}, \mathrm{t})$, then the source function is defined as

$$
S(\mathbf{r}, t)=-\frac{\partial^{2} E(\mathbf{r}, t)}{\partial t^{2}} H(t)-\frac{\partial E(\mathbf{r}, t)}{\partial t} \delta(t)
$$

where $H(t)$ is the Heaviside step function and $\delta(t)$ is the Dirac delta function. The quantity $S(\mathbf{r}, t)$ describes sound source which is determined with its spatial and temporal characteristics.

Linearized equations can be solved easily if we introduce some approximations. We assume that: absorption of the laser light by molecules is instant i.e. the laser pulse is short enough; all excited molecules deexcite through a nonradiative channel. Because gas sample used in the experiment is optically thin and laser beam divergence is neglected, sound source has a cylindrical symmetry. In order to solve the wave equations for known source function and defined initial and boundary conditions, there are two methods: (a) Fourier transform and (b) Green's functions method.

The Fourier transform method can be used in the case of a simple spatial profile of the laser beam and an exponential decay of the excitation energy. The shape of theoretical PA signals, which is obtained by solving wave equation using Fourier method for three simple source functions end exponential decay of the excitation energy, we can show in the form $[1,9]$ :

$$
\delta p\left(r^{*}, t^{*}\right)=\frac{R E_{0}}{C_{v} V} \varepsilon \int_{0}^{\infty}\left(l^{2}+\varepsilon^{2}\right)^{-1}\left(-\varepsilon \exp \left(-\varepsilon t^{*}\right)+l \sin l t^{*}+\varepsilon \cos l t^{*}\right) J_{0}\left(l r^{*}\right) h(l) l d l
$$

Quantities $r^{*}$ and $t^{*}$ are dimensionless known as the reduced coordinate and the reduced time. Reduced coordinate is defined as

$$
r^{*}=\frac{r}{r_{L}}
$$

where $r$ is the radial coordinate, $r_{\mathrm{L}}$ is the laser beam radius. Reduced time can be written as $t^{*}$ $=t / \tau_{\mathrm{p}}$, where $t$ is the time, $\tau_{\mathrm{p}}$ is sonic transit time $\left(\tau_{\mathrm{p}}=\mathrm{r}_{\mathrm{L}} / c\right), c$ is the speed of sound, $R$ is the universal gas constant, $E_{0}$ is absorbed energy, $V$ is the irradiated volume, $C_{\mathrm{V}}$ is the molar heat capacity at constant volume, $l=k r$, is a dimensionless quantity ( $k$ is the wave vector). $J_{0}$ and $J_{1}$ are the first-kind Bessel functions of orders 0 and 1. Dimensionless parameter

$$
\varepsilon=\frac{\tau_{p}}{\tau_{V-T}}=\frac{r_{l}}{c \tau_{V-T}}
$$

is relevant in this study, because, the shape and intensity of PA signals depend on the quantity $\varepsilon$ and this quantity depends on the relaxation time. Knowing the PA signal shape, it is possible to calculate the relaxation time of excited molecules. Function $h(1)$ depending on the energy spatial profile. For some simple cases, this function has the form of

$$
\begin{gathered}
h_{G}(l)=\frac{1}{2} \exp \left(-\frac{1}{4} l^{2}\right) \text { (Gauss profile) } \\
h_{t h}(l)=J_{1}(l) / l \text { (top hat profile) } \\
h_{L}(l)=K_{0}\left(\frac{1}{2} l\right) \text { (Lorentz profile) }
\end{gathered}
$$


where $J_{1}$ is the first kind Bessel function of order 1 and $K_{0}$ is the modified Bessel function of zero order.

In this paper we will calculate theoretical PA signals using Fourier method (3), for three simple laser beam profile given by relations (6a), (6b) and (6c). We assume that function which describes decay of the excitation energy is exponential and has a form:

$$
T(t)=H(t) \exp \left(-t / \tau_{V-T}\right)
$$

Green's functions method is far more powerful in dealing with arbitrary source functions. This method allows us to calculate the photoacoustic signal for arbitrary laser beam profile and excitation energy decay. The quantity $E(\mathrm{r}, \mathrm{t})$ in relation (2) is energy density and it is very significant in solving the equations, because its temporal evolution, in some cases, is independent of the coordinates, so this quantity is factorable into spatial and temporal parts. Spatial parts $R(\mathrm{r})$ is determined by the geometric characteristics of laser radiation and the absorption characteristics of medium and $T(\mathrm{t})$ is temporal parts of the energy density determined by relaxation properties by excited molecules. Based on previous research we can conclude that the spatial profile of the laser beam affects on the measurement accuracy of the vibrational-to-translational relaxation time. Determining the laser beam profile $R(\mathrm{r})$ from known PA signals is inverse problem. Possible solution for the more accurate reconstruction of the laser beam profile gives the back-projection method used in photoacoustic tomography (PAT). The quantity which is directly obtained in PAT is the density of the absorbed energy. For a homogeneous distribution of the radiation and for the homogeneous absorber (gas mixture) the absorbed energy density corresponds to the radiation distribution. The spatial profile of the laser beam, representing the radiation distribution. Using time-domain back-projection algorithm laser beam spatial profile $R(\mathrm{r})$ and relaxation time $\tau_{\mathrm{V}-\mathrm{T}}$, can be obtained simultaneously. The idea of simultaneous determination of these values is derived from the needs to solve the problem where we have two unknowns quantities $\left(r_{\mathrm{L}}\right.$ and $\tau_{\mathrm{V}-\mathrm{T}}$ ) related to equation (5). To solve problems, relaxation time $\tau_{\mathrm{V}-\mathrm{T}}$ was treated as a known quantity. In practice it is usually unknown. In fact, we have to know the laser beam profile to determine it by photoacoustics. On the other hand, we have to know $\tau_{\mathrm{V}-\mathrm{T}}$ to determine the beam profile [3]. This method and method described in previous article [8] exactly reconstruct the profile. However both methods require more time for calculation because the simultaneous determination of $R(r)$ and $\tau_{\mathrm{V}-\mathrm{T}}$ include the procedures that has to be iteratively repeated ( $\tau_{\mathrm{V}-\mathrm{T}}$ values are used to calculate the laser beam profile, and obtained profile has to be used to calculate $\tau_{\mathrm{V}-\mathrm{T}}$ again). On the contrary, our method described bellow based on computational intelligence calculate the laser beam profile and relaxation time $\tau_{\mathrm{V}-\mathrm{T}}$ instantaneously and with high accuracy.

\section{Neural Networks Based Determination of the Laser BeAm Profile AND VIBRATIONAL-TO-TRANSLATIONAL RELAXATION TIME BY PULSED PHOTOACOUSTICS}

Since calculations of $\tau_{\mathrm{V}-\mathrm{T}}$ are usually time consuming, complex and computationally demanding processes, far away from the real-time regime, to overcome these obstacles, a real-time calculation method based on the neural computation can be proposed.

Artificial neural networks (ANNs) are highly parallel connectionist systems modeled on biological neurons $[10,11]$. However, the models most commonly used are far simpler than their biological counterparts. The key features of each of these simulated neurons are the in- 
puts, the activation function, and the outputs. The computational power in an ANN is derived from the density and complexity of the interconnections. A simple ANN is composed of several layers, the input layer, the number of hidden layers and the output layer. Between the layers of units are connections containing weights. These weights serve to propagate signals through the network. Typically, the network is trained using a technique which can be thought of as gradient descent in the connection weight space. In order to find the weights which produce correct outputs for given inputs, the most commonly used method for weight modification is error back propagation. Once the network has been trained, given any set of inputs which are sufficiently similar to those on which it was trained, it becomes capable of reproducing the associated outputs by propagating the input signal forward through each connection until the output layer is reached. ANN models nowadays are considered as use computational intelligence for classification, pattern recognition and prediction.

Here, we have employed feed forward multilayer perception networks which are trained in an offline batch training regime to estimate simultaneously $\mathrm{R}(\mathrm{r})$ and $\tau_{\mathrm{V}-\mathrm{T}}$ from a given photoacoustic signals. The ANN used in this study was a standard back propagation neural network with three layers: an input layer, a hidden layer and an output layer. Network inputs are $n$ equidistant points on a $t^{*}$ axis in which given photoacoustic signal is sampled. Here we have presented results for trials in which neural network had 21 inputs, i.e. PA signal was sampled in 21 points. Outputs of the neural network are value of $\varepsilon$, estimate of $r^{*}$ and profile shape class. Variables $\varepsilon$ and $r^{*}$ are defined with equations (5) and (4) while profile shape class distinguishes Gauss, top hat and Lorentz profile shapes.

Thus, the input network layer consisted of 21 input neurons, and the output layer consisted of three output neurons, while the single hidden layer consisted of 15 hidden neurons (Figure 1). The number of the hidden network layers and the number of neurons in the hidden layer were determined through trial-and-errors process.

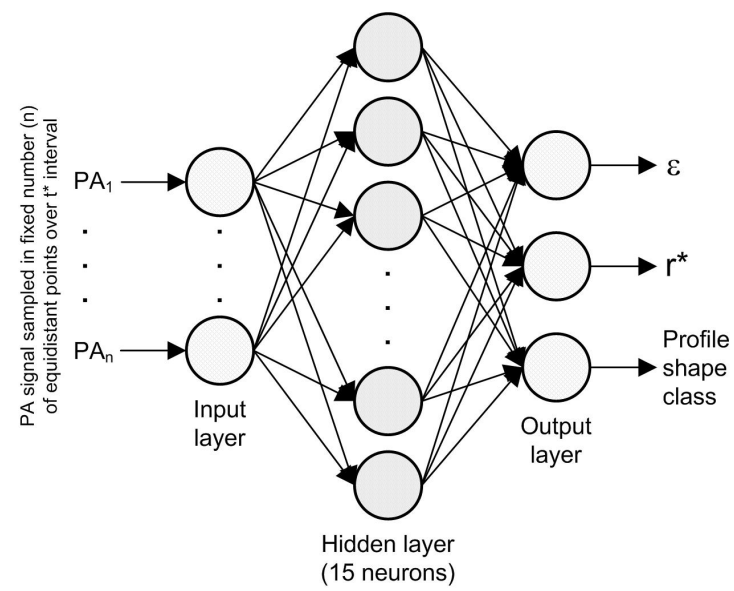

Fig. 1 Neural networks application adopted for the real-time photoacoustic measurements.

For training, the back propagation Levenberg-Marquardt algorithm was used, while the mean squared error was used as performance measure during training. The network training, validation and testing data sets were randomly selected from the same data set of 120 PA signals. Those PA signals were calculated by Fourier method formula (3) and 40 
signals were constructed for each Gauss (6a), top hat (6b), and Lorentz (6c) profile shape. Those shapes were assigned class numbers 1,2 and 3 respectively. For each profile shape, $\mathrm{r}^{*}$ was taking values $39,40,41$ and 42 and for each $r^{*}$ value $\varepsilon$ was varied in ten values: $0.5,1,1.5,2,2.5,3,3.5,4,4.5$ and 5 . The data set of described 120 PA signals used for network training, each sampled in 21 equidistant points, is shown in Figure 2.

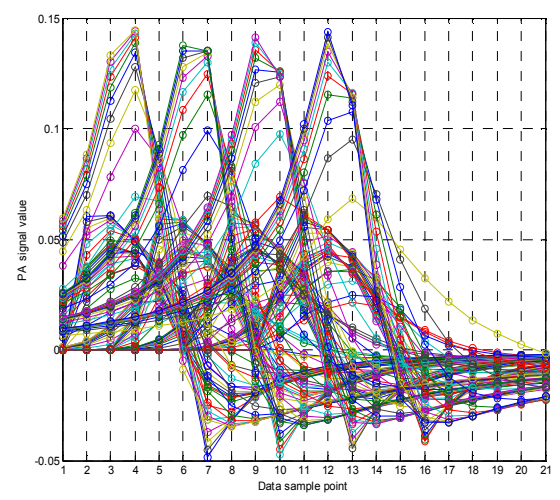

Fig. 2 Data set of 120 PA signals with three profile shapes, four values of $r^{*}$ ranging in $[39,42]$ and ten $\varepsilon$ values ranging in $[0.5,5]$ where signals were sampled in 21 points.

Training sample (84 PA signals) was presented to the network during training, and the network was adjusted according to its error; validation sample (18 PA signals) was used to measure network generalization, and to halt training when generalization stopped improving; finally, the testing sample (18 PA signals) had no effect on training and so provided an independent measure of network performance during and after training.

ANN training based on the mean squared error as performance measure during training is presented in Figure 3.

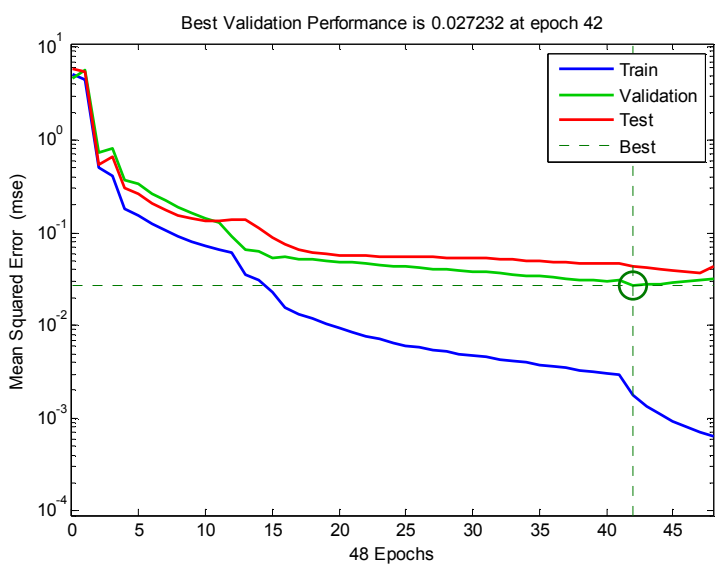

Fig. 3 Artificial neural network training based on MSE

This method could be efficiently tested by simulation, i.e. by searching known parameters of the calculated theoretical photoacoustic signal. Such simulation tests have 
confirmed that presented methodology finds simultaneously laser beam profile and vibrational-to-translational relaxation time efficiently and with good precision. The most important is that once the training phase is over in the application phase ANN provides determination of the laser beam profile and vibrational-to-translational relaxation time by pulsed photoacoustics practically instantaneously, which means that this method easily provides real time operation.

Obtained results indicate that if sufficient amount of simulations and/or experimental data are provided, neural networks are capable of performing the given task efficiently.

\section{Application of Genetic Algorithms for the ObServed Problem}

Genetic algorithms are one of the evolutionary computational intelligence techniques inspired by Darwin's theory of biological evolution. They were proposed in 1975 by Holland [12] and have later been analyzed and explained by Goldberg [13]. Genetic algorithm has three major applications: intelligent search, optimization and machine learning and is a framework for solving various types of the problems. First a corresponding representation of a problem in a genetic world has to be found, then a starting population of candidate descriptions has to be created and finally the evolution takes care of producing better solutions. GAs provide solutions using randomly generated bit strings (chromosomes) for different types of problems, searching the most suitable among chromosomes that make the population in the potential solutions space. The GAs process itself is a simple loop that always follows the same way - use of genetic operators and selection of objects for reproduction. GAs have been proven capable of solving large scale or complex problems and they are commonly used as search mechanism when direct search is impossible. Genetic optimization is an alternative to the traditional optimal search approaches which make difficult finding the global optimum for nonlinear and multimodal optimization problems. GA maintains and manipulates a population of solutions and implements a survival of the fittest strategy in its search for better solutions. The fittest individuals of any population tend to reproduce and survive to the next generation thus improving successive generations.

Implementation of the GA requires the determination of six fundamental issues: chromosome representation, selection function, genetic operators, initialization, termination and evaluation function. Chromosome representation scheme determines how the problem is structured in the GA and also determines the genetic operators that are used. Each individual or chromosome is made up of a sequence of genes. Among various types of representations of an individual or chromosome, real-coded representation stands out for its efficiency and precision with more consistent results [14]. Also, real coding makes it possible to use large and even unknown domains for variables.

Selection of individuals plays a significant role in a GA since it determines which of the individuals will survive and move on to the next generation. A probabilistic selection is performed based upon the individual's fitness so that the superior individuals have better chances to be selected. There are several schemes for the selection process: roulette wheel selection and its extensions, scaling techniques, tournament, normal geometric, elitist models and ranking methods.

The basic search mechanism of the GA is provided by the two types of operators: crossover and mutation, which are used to produce new solutions based on the existing 
solutions in the population. Crossover takes two individuals as parents and produces two new individuals while mutation alters one individual to produce a single new solution. An initial population is needed to start the GA procedure. The initial population can be randomly generated or can be taken from other methods.

Here we present the application of genetic optimization for solving of the observed problem of simultaneous $R(r)$ and $\tau_{\mathrm{V}-\mathrm{T}}$ determination by pulsed photoacoustics. Real coded genetic algorithms (GA) $[10,14]$ are used and the problem is solved by fitting the experimental photoacoustic signal with the best matching theoretical one. The computational flowchart of the real coded genetic algorithm (RCGA) optimization process employed in this paper is shown in Figure 4. The GA moves from generation to generation until a stopping criterion is met. The stopping criterion is maximum number of generations, population convergence criteria and lack of improvement in the best solution over a number of generations or target value for the objective function.

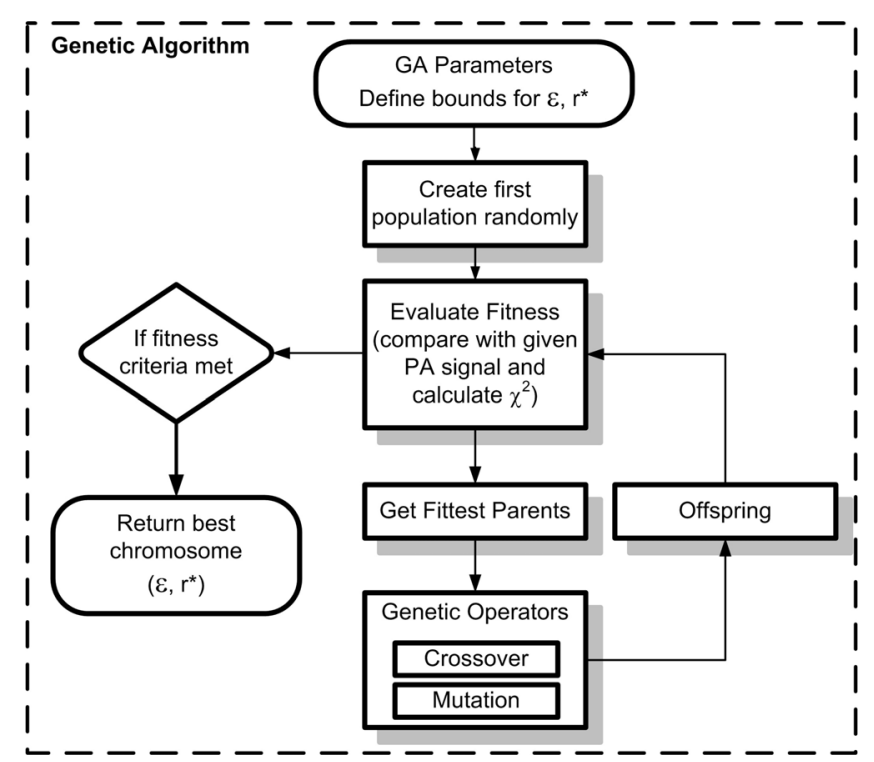

Fig. 4 Real coded genetic algorithm for solving problem of simultaneous $R(r)$ and $\tau_{\mathrm{V}-\mathrm{T}}$ determination by pulsed photoacoustics

The aim is to find combinations of photoacoustic signal parameters, namely radius of the laser beam $r_{\mathrm{L}}$ and $\tau_{\mathrm{V}-\mathrm{T}}$, that provide the best match with the given signal. To accomplish the task, the problem is, as with neural networks, transformed to equivalent search for parameters $\varepsilon$ defined with (5), $r^{*}$ defined with (4) and profile shape class.

In this study, a range for $r^{*}$ was selected as $[20,50]$ and $\varepsilon$ was bounded in the interval $[0.5,5]$. For each individual in the population, i.e. for each $\left(r^{*}, \varepsilon\right)$ pair, a calculated photoacoustic signal with a known profile is determined and compared with experimental signal, so the fitness is defined as the sum of the square deviations $\chi^{2}$ representing deviation of the given and fitted signal. Fitness function is minimized by means of genetic optimization, and in that way the genetic algorithms are used to simultaneously estimate $r_{\mathrm{L}}$ 
and $\tau_{\mathrm{V}-\mathrm{T}}$ that best match given experimental signal. Genetic optimization can be performed separately for different theoretical profile shapes, i.e. for Gauss, top hat and Lorentz profiles. After that obtained best matches are compared, resulting in the single overall theoretical photoacoustic signal best matching the given experimental one. In that way desired parameters $r^{*}, \varepsilon$ and profile shape are efficiently obtained.

In Figure 5 typical GA run in search for photoacoustic signal parameters is presented. The unknown parameters for the Gauss profile shape were found after 40 generations, and the solution was $r^{*}=40$ and $\varepsilon=1.9$.
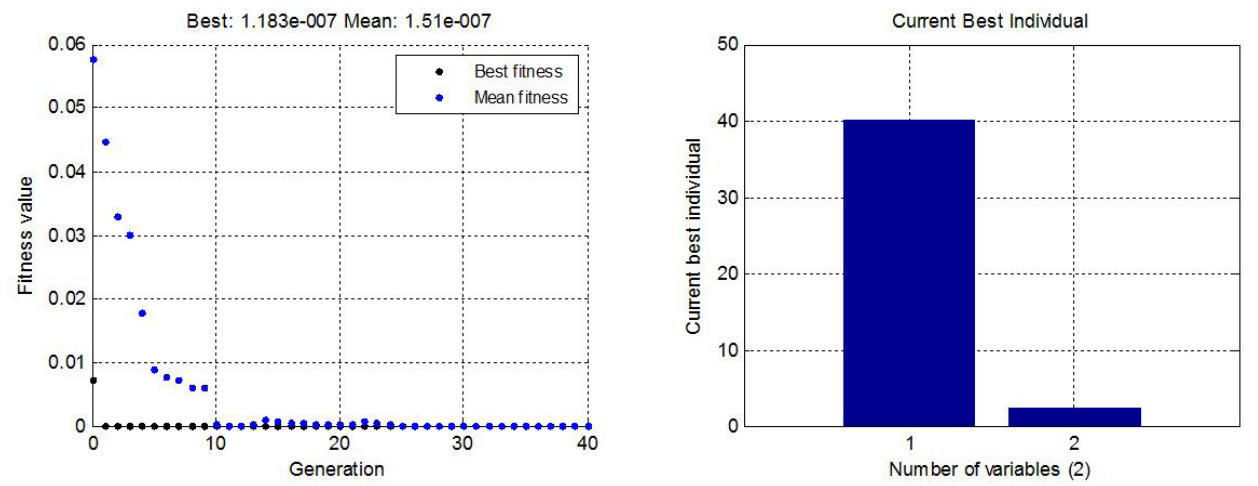

Fig. 5 Typical GA run in search for Typical GA run in search for PA signal parameters.

As with neural networks, this method could be efficiently tested by simulation. Namely, instead of search for unknown parameters that are best matching experimental photoacoustic signal, the method was tested by searching known parameters of the theoretical photoacoustic signal. Such simulation tests have confirmed that presented methodology finds simultaneously laser beam profile and vibrational-to-translational relaxation time efficiently and with good precision.

Compared to the previous methods, presented method is simpler, covers wider parameters' space and requires less time to compute.

\section{CONCLUSION}

Computational intelligence has been applied in different fields of science [15]. In this article we presented the methods based on the photoacoustic signal temporal shape analysis which employ computational intelligence to simultaneously calculate the symmetric laser beam spatial profile and molecular vibrational-to-translational relaxation time. These methods have been tested using theoretical signals obtained by solving wave equations for Gauss, top hat and Lorentz profile.

Results presented in this paper show that we can detect unknown laser beam spatial profile and parameters of PA signals from known simulated (or measured) PA signals, using multilayer feed-forward perception network. Advantages of the ANNs method are real-time operation that provides laser beam spatial profile and relaxation time with high precision, allowing us to more efficiently use in trace gas monitoring with the opportunity 
of application for higher intensities of radiation. Although other presented method that employs genetic algorithms for simultaneously estimate $r_{\mathrm{L}}$ and $\tau_{\mathrm{V}-\mathrm{T}}$ does not operate in real time, it can be considered as an important method that ensuring high accuracy in determining aforementioned quantities and requires less time to compute.

\title{
REFERENCES
}

1. K.M. Beck, A. Ringwelski and R.J. Gordon, Time-resolved optoacoustic measurements of vibrational relaxation rates, Chemical Physics Letters, 121 (6), 529-534 (1985).

2. K.M. Beck and R.J. Gordon, Theory and application of time-resolved optoacoustics in gases, Journal of Chemical Physics, 89 (9), 5560-5567 (1988).

3. M.D. Rabasović, J.D. Nikolić and D.D. Markushev, Simultaneous determination of the spatial profile of the laser beam and vibrational-to-translational relaxation time by pulsed photoacoustics, Applied Physics $B, 88$ (2), 309-315 (2007).

4. M.D. Rabasović, D.D. Markushev and J. Jovanović-Kurepa, Pulsed photoacoustic system calibration for highly excited molecules, Measurement Science and Technology, 17 (11), 1826-1837 (2006).

5. M.D. Rabasović, J.D. Nikolić and D.D. Markushev, Pulsed photoacoustic system calibration for highly excited molecules: II. Influence of the laser beam profile and the excitation energy decay, Measurement Science and Technology, 17 (11), 2938-2944 (2006).

6. S. Mallidi and S. Emelianov, Photoacoustic technique to measure beam profile of pulsed laser systems, Review of Scientific Instruments, 80 (5), 054901 (2009).

7. M. Xu and L.V.Wang, Time-domain reconstruction for thermoacoustic tomography in a spherical geometry, IEEE Transactions on Medical Imaging, 21 (7), 814-822 (2002).

8. M.D. Rabasović and D.D. Markushev, Laser beam spatial profile determination by pulsed photoacoustics: exact solution, Measurement Science and Technology, 21 (6), 065603 (2010).

9. R.T. Bailey, F.R. Cruickshank, R. Guthrie, D. Pugh and I.J.M. Weir, Short time-scale effects in the pulsed source thermal lens, Molecular Physics, 48 (1), 81-95 (1983).

10. M.H. Hassoun, Fundamentals of artificial neural networks, MIT Press, Massachusetts, 1995.

11. J.A. Anderson, An introduction to neural networks, MIT Press, Massachusetts, 1995.

12. J.H. Holland, Adaptation in natural and artificial systems, MIT Press, Massachusetts, 1992.

13. D.E. Goldberg, Genetic algorithms in search, optimization and machine learning. Addison-Wesley Longman Publishing Co., Boston, 1989.

14. F. Herrera, M. Lozano and J.L. Verdegay, Tackling real-coded genetic algorithms, operators and tools for behavioural analysis, Artificial Intelligence Review, 12 (4), 265-319 (1998).

15. Ž.M. Ćojbašić, V.D. Nikolić, I.T. Ćirić and Lj.R. Ćojbašić, Computationally intelligent modelling and control of fluidized bed combustion process, Thermal science journal, 15 (2), 321-338 (2011).

\section{PRIMENA VEŠTAČKE INTELIGENCIJE ZA ISTOVREMENO ODREĐIVANJE PROSTORNOG PROFILA LASERSKOG SNOPA I VIBRACIONO-TRANSLACIONOG RELAKSACIONOG VREMENA IMPULSNOM FOTOAKUSTIKOM}

\author{
Mladena Lukić, Žarko Ćojbašić, Mihailo D. Rabasović, Dragan D. Markushev, \\ Dragan M. Todorović
}

U ovom radu je istražena primena veštačke inteligencije na istovremeno određivanje prostornog profila laserskog zraka i vibraciono-translacionog relaksacionog vremena impulsnom fotoakustikom. Naime, rezultati dobijeni korišćenjem neuronskih mreža i genetske optimizacije su predstavljeni kroz primenu višeslojnih neuronskih mreža i realno-kodiranih genetskih algoritama. Višeslojna neuronska mreža sa prostiranjem signala unapred obučavana je u režimu nadgledanog obučavanja da istovremeno u realnom vremenu da procenu prostornog profila laserskog snopa $R(r)$ (klasu oblika 


\section{M. LUKIĆ, Ž. ĆOJBAŠIĆ, M. D. RABASOVIĆ, D. D. MARKUSHEV, D. M. TODOROVIĆ.}

profila) $i$ vibraciono-translacionog relaksacionog vremena $\tau_{\mathrm{V}-\mathrm{T}} i z$ datog fotoakustičkog signala. Realno kodirani genetski algoritmi (GA) su korišćeni za izračunavanje $\tau_{\mathrm{V}-\mathrm{T}}$ fitovanjem eksperimentalnog signala teorijskim. Cilj je nalaženje kombinacije parametara fotoakustičkog signala, poluprečnika laserskog snopa $r_{L} i \tau_{\mathrm{V}-\mathrm{T}}$ koji daju najbolje poklapanje sa datim fotoakustickim signalom. Predloženi metod značajno skraćuje vreme potrebno za istovremeno određivanje prostornog profila laserskog snopa i vremena relaksacije a ima i prednosti u tačnijem izracunavanju navedenih veličina. Iako do sada razvijeni metodi određuju laserski profil $i$ relaksaciono vreme dovoljno precizno, metod zasnovan na primeni veštačke inteligencije je mnogo pogodniji za praktične primene, kao što je detekcija atmosferskih zagađivača u realnom vremenu.

Ključne reči: fotoakustička spektroskopija, profil laserskog snopa, vibraciono-translaciono relaksaciono vreme, neuronske mreže, genetski algoritmi. 